So sind die Hyylrate von Kali, Kalk, Nickel, Zink u. s. w. aus 1 At. Wasser und 1 At. Sauerstoff auf 2 At. Base zusammengesetzt; das Sesquioxyd des Eisens enthält $\mathbf{3}$ At. Wasser, 3 At. Sauerstoff und 2 At. Metall. Die Hydrate der Binoxyde von Vanadium, Zinn u. s. w. enthalten 2 At. Wasser, 2 At. Sauerstoff und 1 At. Base. Das zweite Oxyd des Baryts ist das einzige bekannte Beispiel, dass das Protoxyd eines Metalls mit 2 Aequivalenten Wasser sich verbindet zu einer Zusammensetzung von 2 At. Wasser, 1 At. Sauerstoff und I At. Base. Der Baryt gleicht auch dem Kali und Natron darin, dass er Wasser bei der Rothglühhitze zurückhält, und nicht dem Kalk, Strontian und der Magnesia,' welche in hoher Temperalur alles damit verbundene Wassers verlieren.

\title{
Ueber die Darstellung des Gerbestoffs;
}

\author{
von \\ Leconnet, \\ Gehülfe in der Centralapotheke in Paris.
}

(Journ. de pharmac. XXII. 149. Auszug.)

Folgendes Verfahren habe ich zur Darstellung des Gerbestoffs am anwendbarsten gefunden. In ein weitmündiges Glas bringe ich Galläpfelpulver und mische dieses mittelst eines bölzernen Spatels mit so viel Aether, nicht mehr, als nölkig ist, un einen Teig zu machen, verstopfe das Glas mit einem Kork, und lasse das Ganze 24 Stunden in Berührung. Hierauf bringe ich das Galläpfelpulver auf ein Stïck starker Leinwand, welches nicht grösser ist, uls das Pulver einzuhüllen, vertheile dieses darauf, und bringe es nach Einschlagen unter die Presse, und. erhalte durch deren Wir- 
kung eine syrupsartige Flüssigkeit. Der Rückstand des Galläpfelpulvers wird auf dieselbe Weise mit Aether behandelt, so oft bis endlich die Ausbeute nicht mehr lohnend ist.

Ich habe in drei verschiedenen Versuchen auf diese Weise 5oo Grammen Galläpfel mit verschiedenen Quantiläten Aether behandelt, und erhielt:

$\begin{array}{ccc}\text { Galläpfel } & \text { Aether } & \text { Gerbstoff } \\ 500 & 450 & 195 \\ 500 & 520 & 230 \\ 500 & 780 & 300 .\end{array}$

Hiernach sind die Verhältnisse vom Tannin zum Aether:

$$
\begin{aligned}
& 1 \text { Versuch }=1: 2,3 \\
& 2-=1: 2,7 \\
& 3-=1: 2,6 .
\end{aligned}
$$

Es lïsst sich hieraus schliessen, dass, je melr man Aetber anwendet, man um so mehr Tannin erhält, aber dass ein Punkt eintritt, wo man, um eine gegebene Quantität zu erhalten, sehr viel Aether anwenderı muss, wesshalb ich es für vortheilhaft halte, dass man aufböre, wenn man die Hälfte des Gewichts der Galläpfel an Tannin erhalten hat.

Wenn ich diese Resultate mit denen durch Deplacirung erhaltenen vergleiche, so ergiebt sich folgendes: Auf 500 Gram. Galläpfel liess ich 63o Gran. Aether wirken. Ich erhieit die bekannten beiden Schichten von gleichem Volum, ich unterwarf das Ganze der Destillation, erhielt 210 Gram. Áther wieder und 105 Gram:-Gerbestoff; ich gab darauf Wasser auf die Galläpfel und bekam dadurch noch 45 Gram. Gerbstoff; ich hatte also 150 Gram. Gerbestoff erhalten und 420 Gram. Aether verloren. Dieses giebt zwischen Tannin und Aether das Verbältniss von 2,8.

Wenn man bei dem vorstehenden Versuche die Menge des $\Lambda$ ethers rerdoppelt, und man die doppelte Menge Gerbstoff erhalten kann, so ist die Differenz nicht so bedeu- 
tend, dass ein Verfahren viel Vorzüge vor dem andern hälte; aber nur einmal habe ich unter denselbən Umständen ein so schörnes Produkt erhalten, und endlich gelangt man auch nicht dahin, die Galläplel durch Deplacirung zu erschöpfen, weil man nach Pelouze dadurch 35 bis 40 Procent Gerbsioff erhält, und ich durch Maceration 6o Procent erhalten habe und noch mehr hätte erhalten können.

\title{
Ueber die Bereitung des arsenikfreien An- timonmetalls;
}

\author{
von \\ Dr. Wilibald Artus, Privatdocent.
}

Mitgetheilt in der pharmaceutischen Section bei der Versammlung der Naturforscher und Aerzte in Jena.

Das so häufige Vorhandenseyn des Arseniks in dem Antimonmetall und dessen Präparaten erheischt, da dasselbe in pharmaceutisch - medicinischer Hinsicht ein sehr wichtiges Netall ist, indem es die vorzüglichsten und kräftigsten Arzneimittel liefert, nothwendigerweise eine nähere Betrachtung, da die Antimonpräparate, durch jene genannte Verunreinigung den belebten Organismus gefährden könnten.

Bevor ich jedoch zu der Beschreibung der Methode gelange, zufolge welcher das $\Lambda$ ntimonmetall arsenikfrei dargestellt werden kann, werde ich der Uebersicht wegen einige von den Methoden herausheben, nach denen es bisher dargestellt wurde. Früher unterschied man 1) den gemeinen oder vegetabilischen und 2) den eisenhaltigen Spiessglanzkönig.

a) Den gemeinen oder vegetabilischen erhält man, wenn man 4 Theile Schwefelantimonium, 3 Theile rohen Weinstein und $1 \frac{T}{2}$ Theile Salpeter genau vermengt und in klei- 